\title{
Gas flow in microchannels with bends
}

\author{
Sylvanus Yuk Kwan Lee ${ }^{1}$, Man Wong ${ }^{2}$ and Yitshak Zohar ${ }^{1,3}$ \\ ${ }^{1}$ Department of Mechanical Engineering, Hong Kong University of Science and Technology, \\ Clear Water Bay, Kowloon, Hong Kong \\ ${ }^{2}$ Department of Electrical and Electronic Engineering, Hong Kong University of Science and \\ Technology, Clear Water Bay, Kowloon, Hong Kong \\ E-mail: mezohar@ust.hk
}

Received 26 March 2001, in final form 21 August 2001

Published 7 September 2001

Online at stacks.iop.org/JMM/11/635

\begin{abstract}
Bends or curves are unavoidable features in fluidic systems due to design or technology constraints. These fluidic elements in a macrochannel always induce secondary flows, which result in pressure loss in addition to frictional loss. In this paper, this phenomenon is investigated on a microscale where flow separation is not expected to develop. A set of microchannels, with the dimensions $20 \times 1 \times 5810 \mu \mathrm{m}^{3}$, with a $90^{\circ}$ turn at the channel centre, has been fabricated using standard micromachining techniques. Three bend configurations have been tested: miter, curved and double-turn. All the microchannels were integrated with pressure microsensors. Argon gas was passed through the microdevices under an inlet pressure of up to $50 \mathrm{psi}$, and the mass flow rate was measured for all the devices as a function of the driving pressure drop. The flow rate through the channel with the miter bend, a single sharp turn at a right angle, was found to be the lowest.

Pressure distributions along the microchannels were recorded, showing an additional pressure drop across the bends. The largest drop was found in the miter bend with the lowest flow rate. The mass flow rate and pressure measurements indicate that secondary flow could develop in microchannels also due to a bend, contrary to expectations.
\end{abstract}

\section{Introduction}

Microfluidic elements have become major components in many microsystems. They can be used either as active devices such as pumps, valves, jets, amplifiers and switches or as passive devices such as flow conduits. However, very little is known about fluid flow in such microgeometries and unexpected phenomena have been observed when dealing with fluidic microsystems [1]. In almost every microfluidic device, a straight and uniform segment is involved as a part of a more complex geometry. Indeed, fluid flow in microchannels has been the subject of intense experimental and theoretical research not only because of its wide range of applications but also because it is the most convenient tool to test our fundamental understanding of microscale fluid mechanics $[2,3]$. The measured gas flow rate and pressure distributions indicate the development of slip flow conditions [4]. This flow field presents a simple balance between pressure gradient and wall shear stress. In most practical applications, however, microducts are not straight due to required turns, and the

3 Author to whom correspondence should be addressed. area cross section is not constant due to local contraction or expansion sections. An important phenomenon in many fluidic systems is flow separation, which may be geometric, from a sharp corner, or dynamic, as in smooth curved bends. The separation of the flow in a duct always induces secondary flow, which results in pressure loss additional to the frictional loss [5]. This flow behaviour is very common, intentionally or otherwise, and is sometimes accepted because avoiding it is too complicated and expensive. Separation and re-attachment of flow in macrosystems have received significant attention because of their importance in many engineering applications [6]. Only recently has similar research in microsystems been started.

The height of microchannel flows is typically of the order of several micrometres and, thus, the reduced Reynolds number in microsystems is very small. Under such conditions, the flow is expected to be of the Hele-Shaw type with no separation even from sharp corners [7]. Indeed, the velocity field around a $30 \mu \mathrm{m}$ wide obstacle inserted between plates and separated by a $5 \mu \mathrm{m}$ gap exhibited in-plane symmetry of motion with two stagnation points on either 


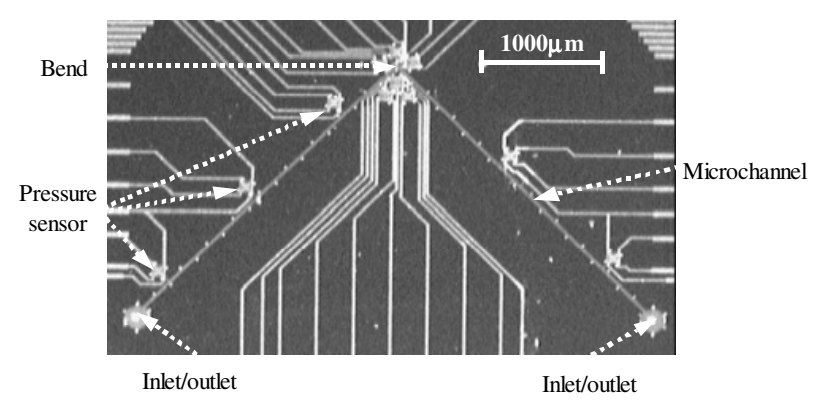

Figure 1. An overall picture of a fabricated device showing a microchannel with a $90^{\circ}$ bend, pressure sensors, channel inlet/outlet and metal interconnects.

side of the cylindrical obstruction with no separation [8]. However, calculations of the shear-driven gas flow in a grooved microchannel demonstrated that, even for a small Reynolds number, the flow had separated and started to recirculate in the grooves [9]. Mass flow rate and pressure distribution measurements in microchannels with a variety of constriction elements [10,11] and contraction/expansion sections [12] have indicated that the pressure loss was significantly higher than the frictional loss of fully attached flow. A possible explanation was the occurrence of flow separation due to the sharp corners.

Although the flow area is still uniform, a bend or a curve in a pipe always induces a loss larger than the simple friction loss due to flow separation at the walls and a swirling secondary flow arising from the centripetal acceleration. This is in contrast to elements such as contraction/expansion or constriction, which change the local streamwise distribution of the flow area. In this paper, microchannels with a variety of bend configurations, of $90^{\circ}$ turn, have been integrated with pressure sensors allowing a detailed investigation of the flow field along the bends.

\section{Device design and fabrication}

The microdevices were surface micromachined on silicon substrates. Each device included a microchannel, with the dimensions $20 \times 1 \times 5810 \mu \mathrm{m}^{3}$. All the microchannels were designed with a $90^{\circ}$ turn mid-way between the inlet and outlet, as shown in figure 1, to eliminate any possible interaction between end effects and bend effects. Microchannels with a variety of bend configurations were fabricated, and the results pertaining to three types are discussed in this paper. Close-up pictures of these bends-miter, curved and double-turn-are shown in figures $2(a)-2(c)$, respectively. In the miter bend the channel turns sharply at a right angle, while in the curved bend the channel turns smoothly with a $60 \mu \mathrm{m}$ radius-of-curvature. In the double-turn bend, the channel turns sharply twice at $45^{\circ}$ with a separation of $50 \mu \mathrm{m}$ between the two bends.

Pressure microsensors were distributed sparsely along the straight sections of the microchannel for reference, and densely around the bending sections. The sensors are based on piezoresistive sensing elements, arranged in a Wheatstone bridge configuration and attached to a membrane, $100 \times 100 \mu \mathrm{m}^{2}$ in size, as seen in figure 2. Any pressure difference across the membrane results in its deflection, thus straining the sensing elements and changing their resistance. The voltage change due to the resistance change is recorded,

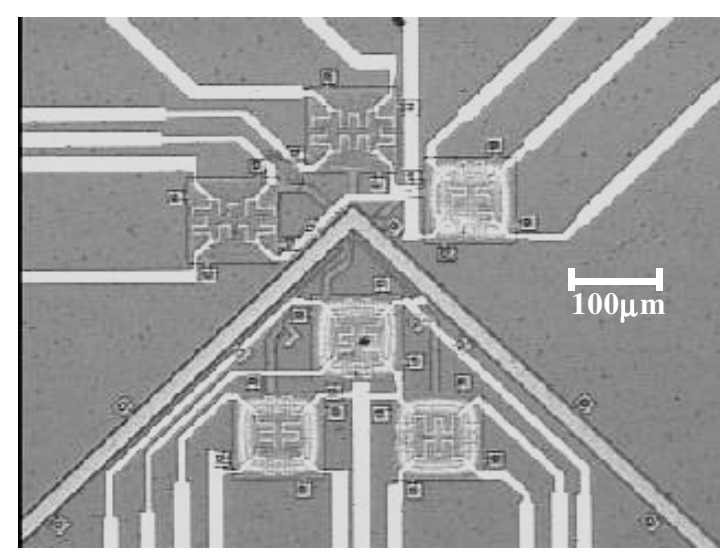

(a)

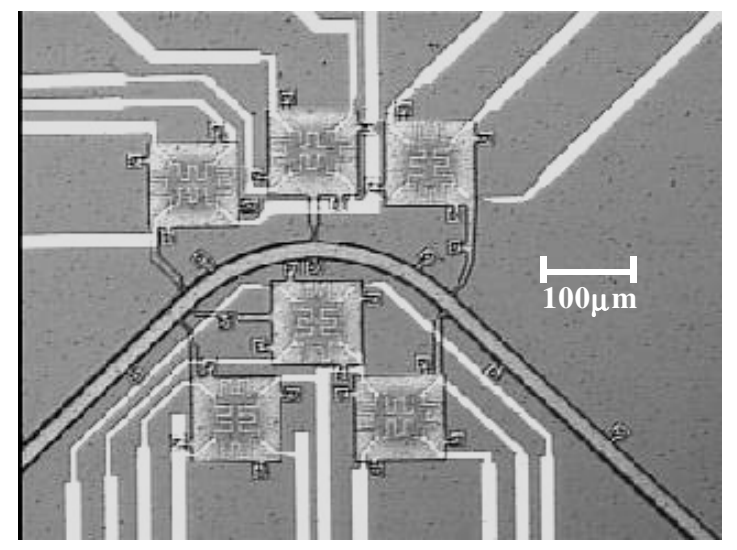

(b)

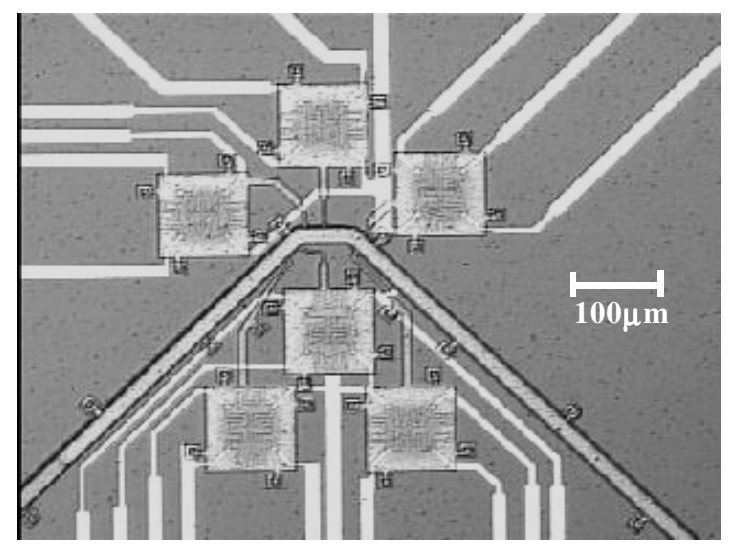

(c)

Figure 2. Close-up pictures of three different bends located at the centre of the $20 \mu \mathrm{m}$ wide microchannels: $(a)$ miter; $(b)$ curved; (c) double-turn.

and the pressure difference can be recovered using a calibration curve. The cavities under the sensor membranes were connected to the microchannel via small capillaries, about 2-4 $\mu \mathrm{m}$ wide, to eliminate the influence of the pressure sensors on the fluid flow [4].

A schematic diagram of the cross sections of the main fabrication steps is shown in figure 3. The fabrication starts with the formation of a $0.2 \mu \mathrm{m}$ thick thermal oxide and a $0.3 \mu \mathrm{m}$ thick low-stress nitride film on a $\mathrm{Si}$ wafer for insulation. A $0.7 \mu \mathrm{m}$ thick low-temperature oxide (LTO) was deposited and patterned to form the channel and sensor 
Gas flow in microchannels with bends

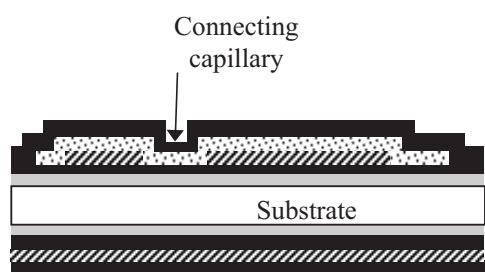

(a)

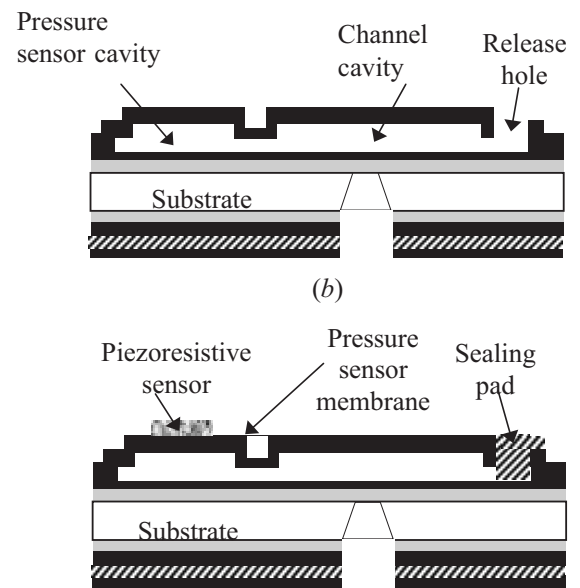

(c)

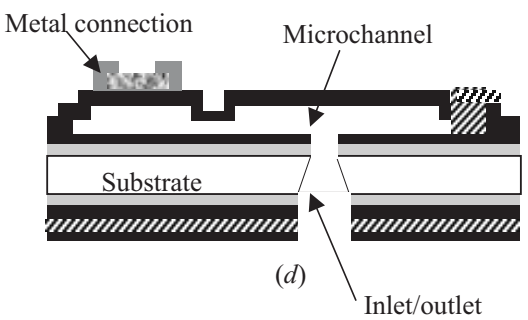

Figure 3. A schematic diagram of the cross sections of the major fabrication steps.

chambers. This was followed by another low-pressure chemical vapour deposition (LPCVD) and patterning of a $0.3 \mu \mathrm{m}$ thick phosphosilicate glass (PSG) film to shorten the etch time of the sacrificial layer. The phosphorous content in the PSG determines the sacrificial layer etching rate. In general, the higher the phosphorous concentration is, the faster the etch rate [13]. In the present work, the phosphorous content was about $6 \%$. The pattern of the PSG layer is similar to that of the LTO layer, except for the distributed pads along the sides of the patterns to be used as etching holes. Next, a structural layer of $1 \mu \mathrm{m}$, low-stress nitride was deposited by LPCVD to form the channel walls and ceiling as well as the sensor membrane (figure 3(a)). Anisotropic etching was used to form the inlet/outlet holes from the back of the wafer, using the oxide/nitride double layer as an etch mask. Following the opening of the etching holes, the sacrificial layer was etched in $49 \%$ hydrofluoric acid (HF) (figure $3(b)$ ). This step was very critical as the release time could not be too long, since concentrated $\mathrm{HF}$ also etches silicon nitride at a rate of $0.04 \mu \mathrm{m} \mathrm{min}^{-1}$. Therefore, the interval distance between adjacent etching holes should be designed properly. In this work, the time required to release all the structures was less than $10 \mathrm{~min}$. The formation of the channel chambers was completed with the deposition of a $0.6 \mu \mathrm{m}$ thick LTO layer

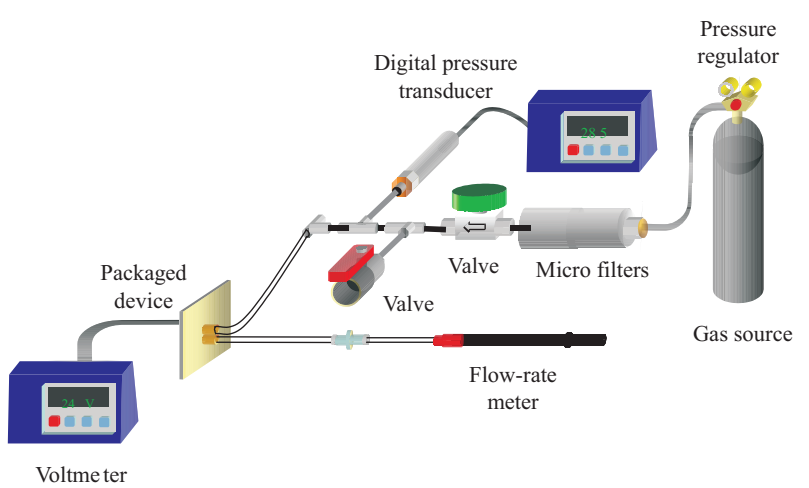

Figure 4. A schematic illustration of the experimental set-up.

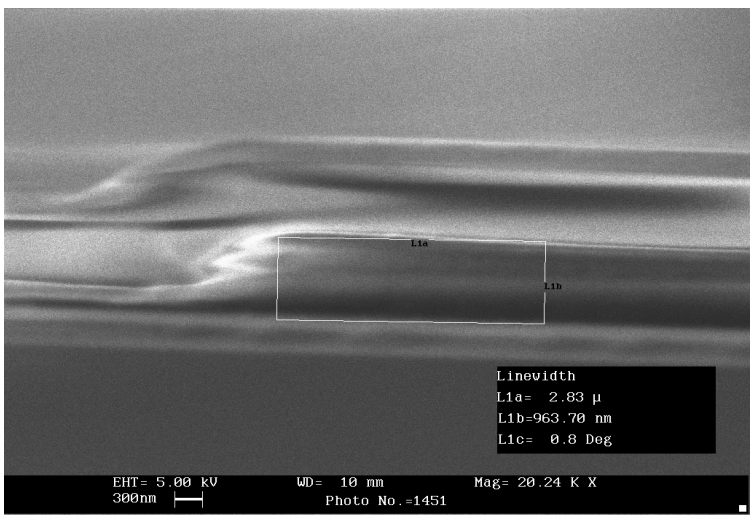

Figure 5. SEM micrograph of a microchannel cross section.

to seal the etching holes. Then, a LPCVD polysilicon layer, $0.5 \mu \mathrm{m}$ in thickness, was deposited, boron-doped and patterned to form the piezoresistors on the sensor membrane (figure 3(c)). The LPCVD process resulted in very low pressure in the chambers, and the diaphragms were deflected downward under the ambient atmospheric pressure. Finally, a $0.8 \mu \mathrm{m}$ thick aluminium layer for interconnections was sputter deposited, patterned and sintered to complete the fabrication process (figure $3(d)$ ).

\section{Experimental set-up}

A schematic diagram of the experimental set-up is shown in figure 4. Argon gas was passed through the microdevices, and the flow rate was measured using a glass syringe with a volume of $10 \mu \mathrm{l}$. The volume flow rate was determined visually as a meniscus of water travelling past the marked scale on the syringe as a function of time. Multiplying this rate by the outlet gas density of $1 \mathrm{~atm}$ yields the mass flow rate. During the sacrificial-layer etch to create the channel cavity, the nitride films forming the upper and lower solid boundaries are also slightly etched. This results in a channel height larger than the sacrificial-layer thickness. Since the flow rate is a cubic function of the channel height, it is very important to determine its value as accurately as possible. Hence, a few microchannels were cut and scanning electron microscopy (SEM) micrographs of the cross sections were taken, as shown in figure 5, to determine the height of the completed microchannels. The average height measured from 


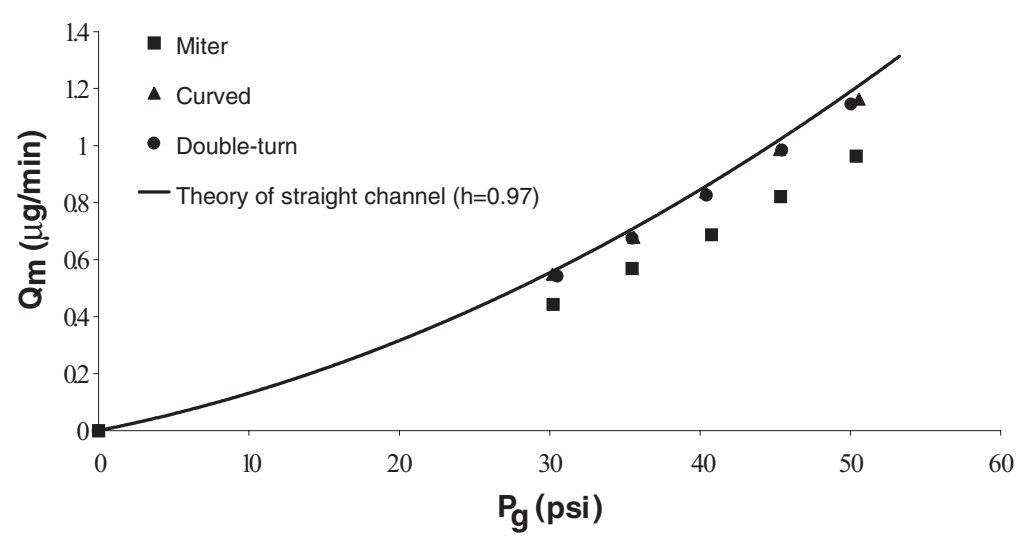

Figure 6. A comparison between the measured mass flow rate for the three microchannels with bends and calculated mass flow rate for a straight microchannel.

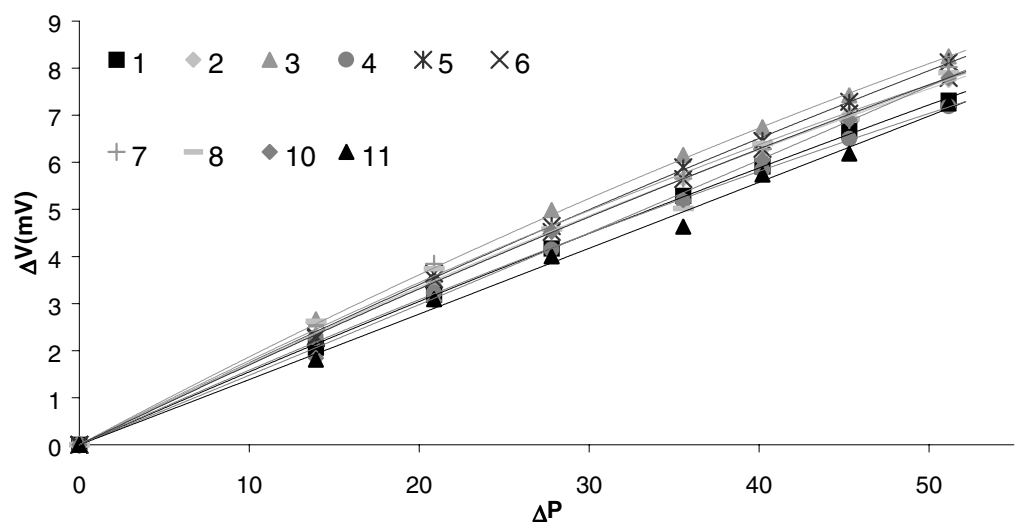

Figure 7. Calibration curves of the pressure sensors of the microchannel with a double-turn bend.

the pictures was found to be about $0.97 \mu \mathrm{m}$, compared with the design height of $1 \mu \mathrm{m}$. Since the tested microchannels were fabricated simultaneously with the microchannels used for the SEM pictures, we assume that the height of all the microchannels is the same. The thin nitride film serving as the channel ceiling could be deflected outward due to the flow pressure during operation; hence, effectively enlarging the channel height. The bulging effect was experimentally found to be negligible for a driving gauge pressure lower than $100 \mathrm{psi}$ [14]. The driving argon pressure in the present experiments did not exceed 50 psi so this effect can be neglected.

\section{Mass flow rate measurements and calculations}

Flow rate measurements were conducted to assess the effect of the bend configuration on the overall fluid flow. However, in order to provide a theoretical reference for this assessment, the flow rate through a straight microchannel is first calculated assuming the flow is fully attached everywhere.

\subsection{Calculations of mass flow rate through a microchannel with a bend}

In contrast to constriction microchannels [11], which are straight but with a non-uniform cross-section area, microchannels with bends are not straight but the cross-section area can be considered uniform. If the flow is fully attached, the mass flow rate for straight and curved microchannels should be the same, provided that the cross-section area is equal. The mass flow rate in a straight and uniform microchannel under a given pressure drop, $\Delta P=P_{\mathrm{i}}-P_{\mathrm{o}}$, is given by [15]:

$$
Q_{\mathrm{m}}=\frac{H^{3} W P_{\mathrm{o}}^{2}}{24 R T L \mu}\left[\left(\frac{P_{\mathrm{i}}}{P_{\mathrm{o}}}\right)^{2}-1+12 K n_{\mathrm{o}}\left(\frac{P_{\mathrm{i}}}{P_{\mathrm{o}}}-1\right)\right]
$$

where $H, W$ and $L$ are the channel height, width and total length from inlet to outlet respectively, $P_{\mathrm{i}}$ and $P_{\mathrm{o}}$ are the inlet and outlet pressure, $T, \mu$ and $R$ are the fluid temperature, viscosity and specific gas constant respectively, and $K n_{\mathrm{o}}=$ $\lambda_{\mathrm{o}} / H$ is the outlet Knudsen number based on the outlet mean free path, $\lambda_{0}$. In the present experiments, $K n_{\mathrm{o}}=0.06$ for argon at an exit pressure of $1 \mathrm{~atm}$. This model accounts for compressible and slip flow effects, but neglects acceleration and non-parabolic velocity profile effects [14]. Mass flow rate calculations based on equation (1) for a microchannel, with the dimensions $20 \times 0.97 \times 5810 \mu \mathrm{m}^{3}$, are shown in figure 6 as a solid curve.

\subsection{Measurements of the mass flow rate}

The mass flow rate of argon, $Q_{\mathrm{m}}$, was measured as a function of the total pressure drop, $\Delta P$, for the channels with the three different bends. The results are compared in figure 6 with the calculated flow rate for the straight microchannel. The measured mass flow rates in all the microchannels with bends 


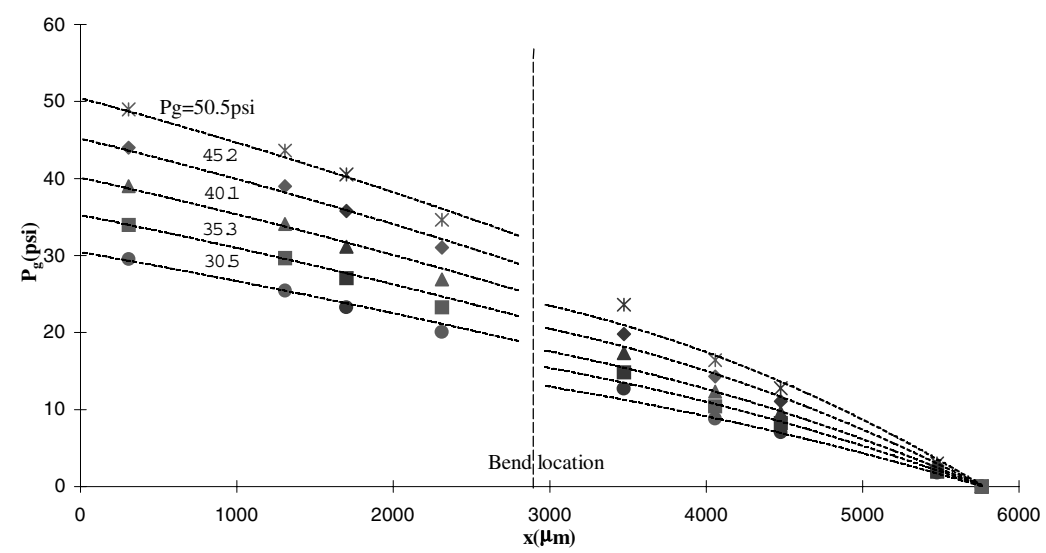

(a)

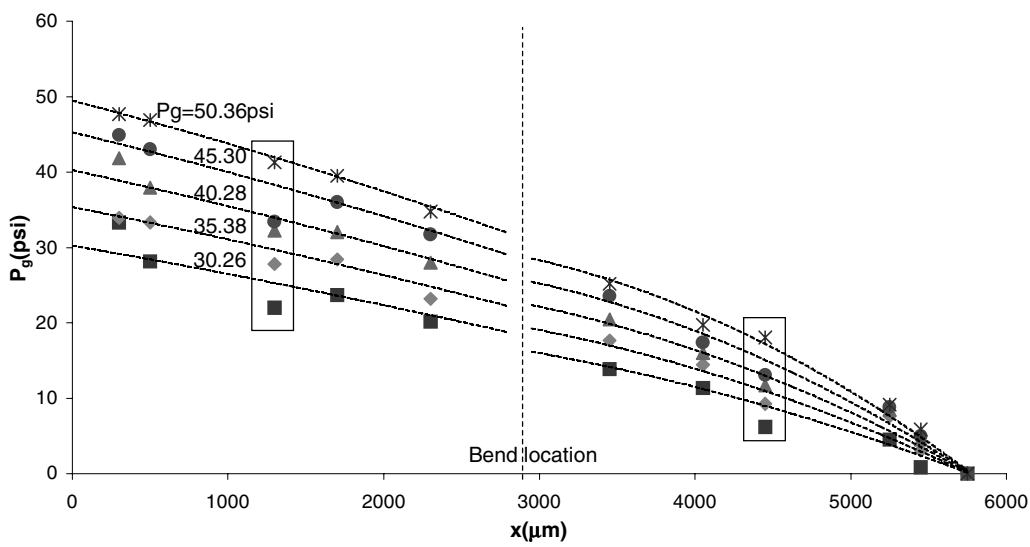

$(b)$

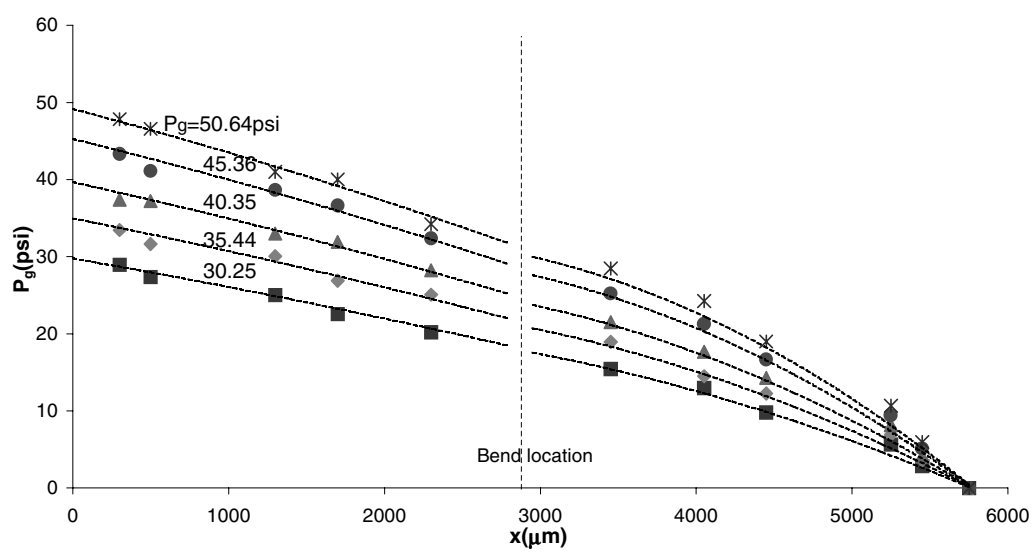

(c)

Figure 8. Streamwise pressure distributions along the microchannels with the $(a)$ miter, $(b)$ curved, and $(c)$ double-turn bends.

are smaller than the analytical values. The flow rate through the miter bend channel is the lowest, about $80 \%$, while the flow rate through either the double-turn or the curved bend channels is about $95 \%$ of the calculated flow rate for a straight channel. Since the cross section is constant, the flow rate through all the devices should be equal to a straight channel flow rate if the flow is fully attached with no separation, so that the only resistance to the flow is due to friction. The lower measured values indicate that the presence of the bends results in additional pressure loss and, subsequently, in a reduction of the flow rate. Furthermore, the loss due to one sharp turn of $90^{\circ}$ (miter) is larger than the loss due to two sharp turns of $45^{\circ}$.
This clearly demonstrates that the overall bend angle, $90^{\circ}$ in all the devices, is less important than the detailed geometry of the bend.

\section{Streamwise pressure distributions}

All the pressure sensors in each device were calibrated prior to the measurements, as in the example shown in figure 7. The sensor sensitivity was, on average, about $0.08 \mathrm{mV} \mathrm{psi}^{-1} \mathrm{~V}^{-1}$, which was sufficient for the pressure range tested in this work. The accuracy of the pressure measurements is estimated to be within $\pm 3 \%$ of the nominal value. 


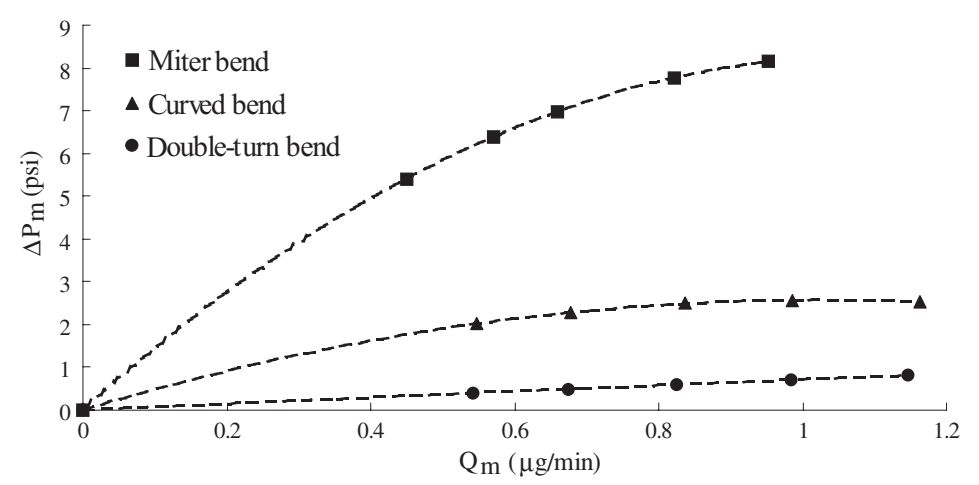

Figure 9. Estimated pressure loss due to the bends as a function of the mass flow rate.

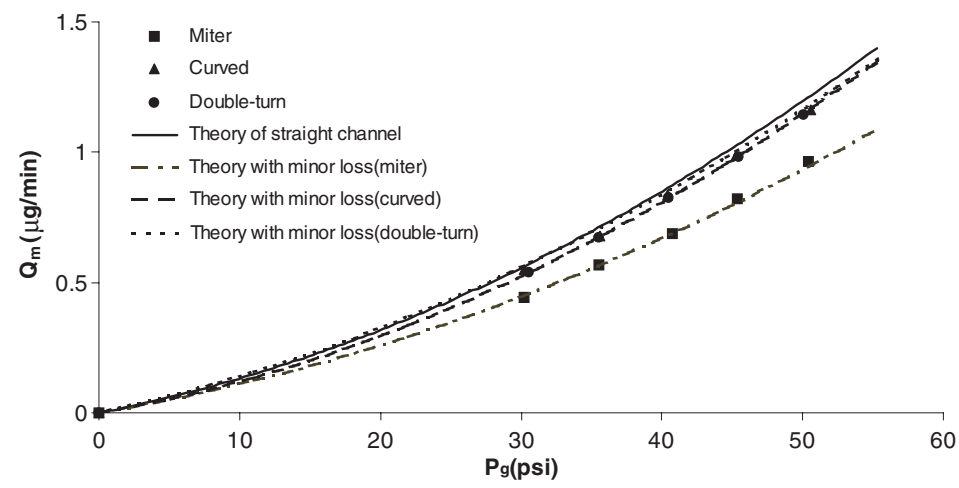

Figure 10. A comparison between the measured and corrected calculations of mass flow rate accounting for pressure loss due to the bends.

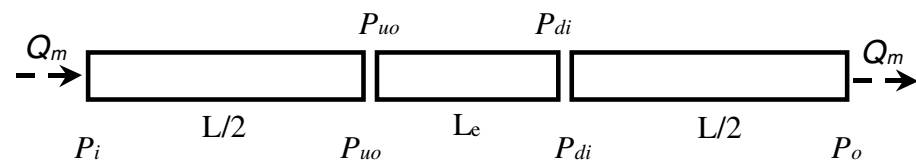

(a)

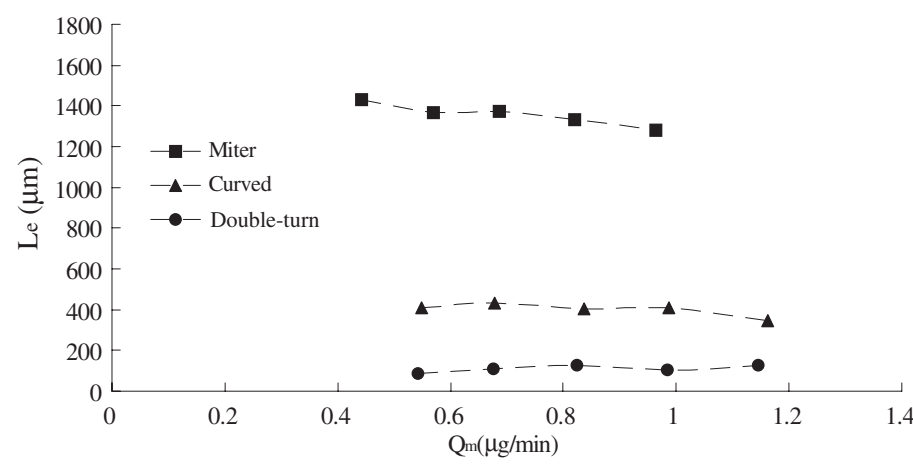

$(b)$

Figure 11. Equivalent length dependence on the mass flow rate: $(a)$ physical model; $(b)$ empirical calculations (equation (4)).

\subsection{Pressure distributions along the straight segments}

In order to investigate the cause of the additional loss, pressure distributions along the three microchannels were recorded. The results for the miter, curved and doubleturn bends are summarized in figures $8(a)-8(c)$, respectively. Unfortunately, not all of the pressure sensors always function properly as indicated by the framed data points in figure $8(b)$. Extrapolating the measured pressure distribution towards the bend, the outlet pressure of the upstream straight section, $P u_{\mathrm{o}}$, and the inlet pressure of the downstream straight section, $P d_{\mathrm{i}}$, can be estimated. The pressure distribution is then calculated for each section, based on the same analytical model used for deriving equation (1) [15]:

$$
\begin{aligned}
& \frac{P(x)}{P u_{\mathrm{o}}}=-6 K n_{\mathrm{uo}}+\left\{\left(6 K n_{\mathrm{uo}}+\frac{P_{\mathrm{i}}}{P u_{\mathrm{o}}}\right)^{2}-\left[\left(\frac{P_{\mathrm{i}}^{2}}{P u_{\mathrm{o}}^{2}}-1\right)\right.\right. \\
& \left.\left.+12 K n_{\mathrm{uo}}\left(\frac{P_{\mathrm{i}}}{P u_{\mathrm{o}}}-1\right)\right]\left(\frac{x_{\mathrm{i}}}{L / 2}\right)\right\}^{1 / 2}
\end{aligned}
$$




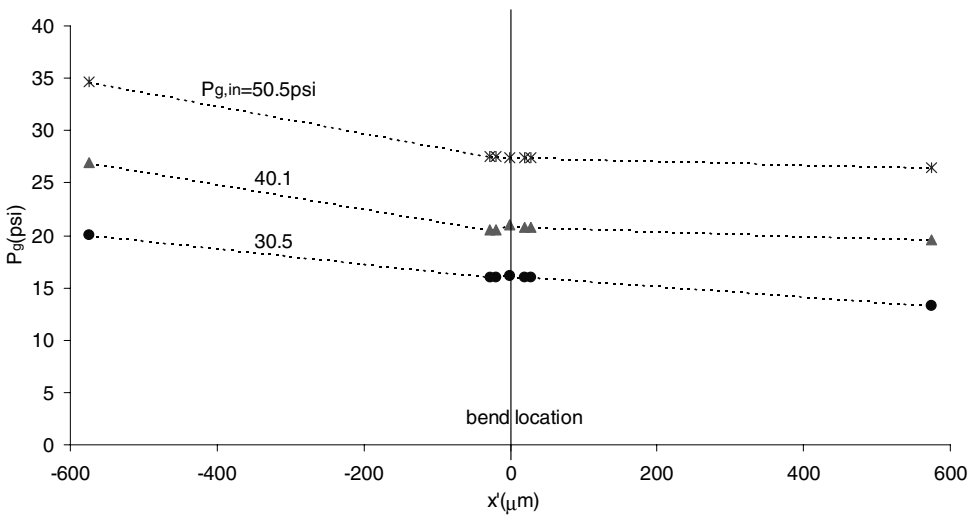

(a)

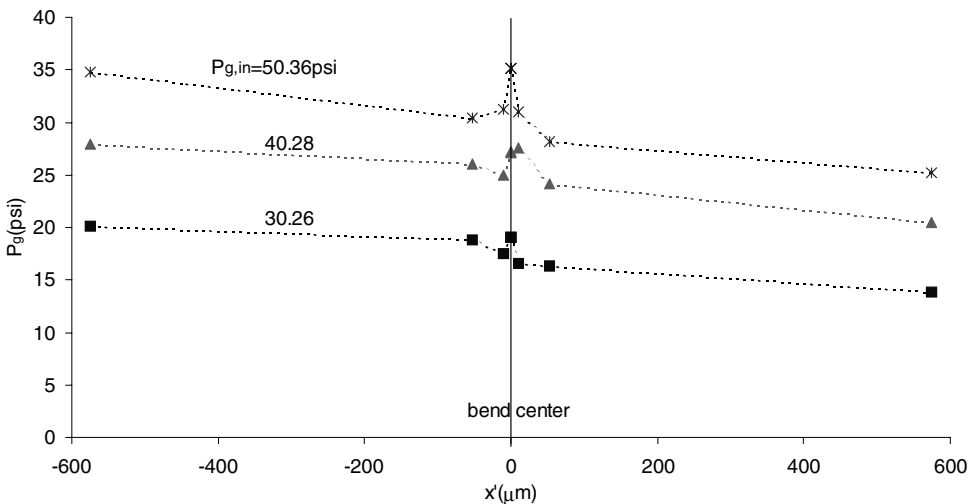

(b)

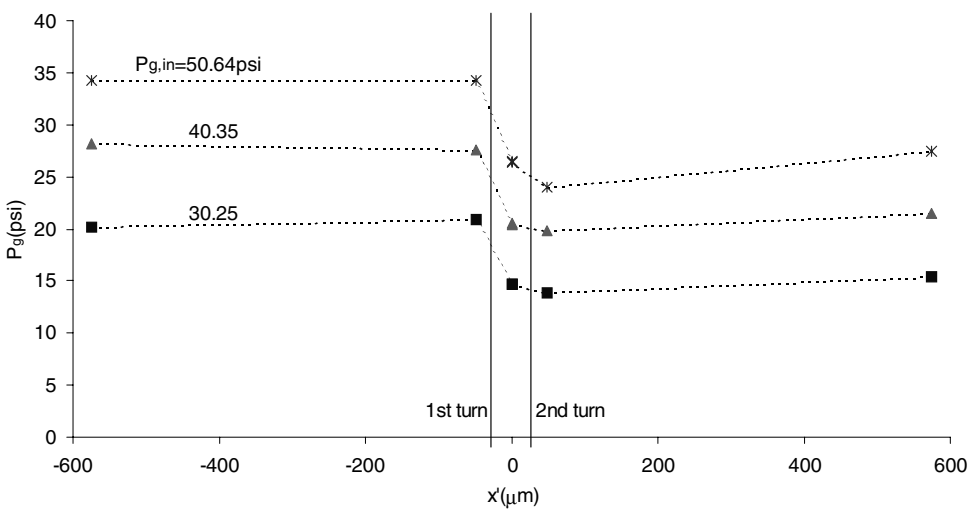

$(c)$

Figure 12. Detailed pressure distributions along the outer wall of the $(a)$ miter, $(b)$ curved, and $(c)$ double-turn bends.

$$
\begin{aligned}
& \frac{P(x)}{P_{\mathrm{o}}}=-6 K n_{\mathrm{o}}+\left\{\left(6 K n_{\mathrm{o}}+\frac{P d_{\mathrm{i}}}{P_{\mathrm{o}}}\right)^{2}-\left[\left(\frac{P d_{\mathrm{i}}^{2}}{P_{\mathrm{o}}^{2}}-1\right)\right.\right. \\
& \left.\left.+12 K n_{\mathrm{o}}\left(\frac{P d_{\mathrm{i}}}{P_{\mathrm{o}}}-1\right)\right]\left(\frac{x_{\mathrm{o}}}{L / 2}\right)\right\}^{1 / 2}
\end{aligned}
$$

where $x_{\mathrm{i}}$ is the streamwise distance from the channel inlet, $x_{0}$ is the streamwise distance from the bend at the channel centre, and $K n_{\text {uo }}=P_{\mathrm{o}} K n_{\mathrm{o}} / P u_{\mathrm{o}}$ is the Knudsen number at the upstream section outlet. The channel length between the inlet/outlet and the bend was designed to be large, about $2900 \mu \mathrm{m}$, to ensure the development of regular channel flow away from the bend such that the end effects can be neglected. Indeed, the calculated nonlinear pressure distributions outside the bend region, shown in figure 8 , agree reasonably well with the measured values. This suggests that the flow development in the straight sections between the inlet/outlet and the bend is similar to that in a straight microchannel.

\subsection{Pressure loss due to the bends}

A distinct drop in the static pressure across the bends is evident in the streamwise pressure distributions plotted in figure 8 . This pressure drop can be estimated, $\Delta P_{\mathrm{m}}=P u_{\mathrm{o}}-P d_{\mathrm{i}}$, and is plotted in figure 9 as a function of the mass flow rate. The pressure drop increases monotonically, though not linearly, with increasing mass flow rate (increasing total pressure drop). Accounting for this additional pressure loss due to the bend, often labelled as a minor loss, the calculated flow rates can be corrected. 


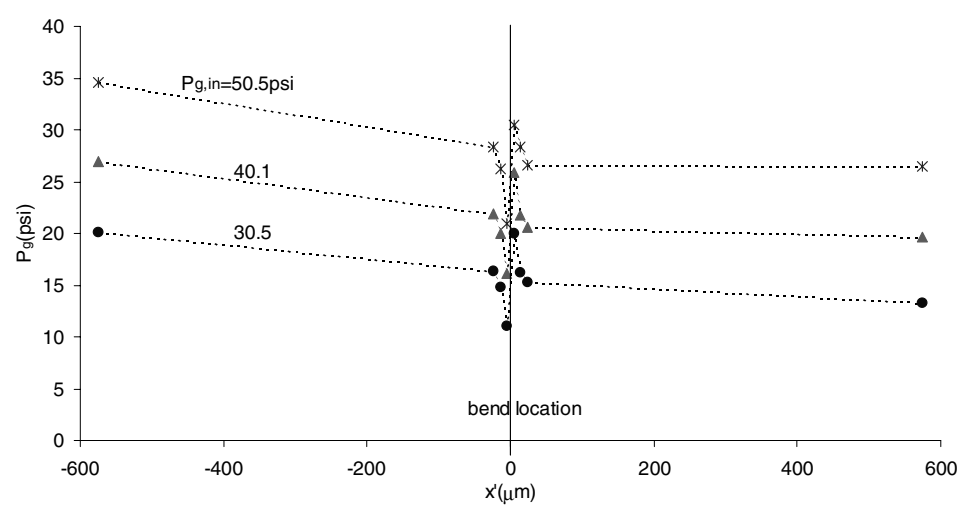

(a)

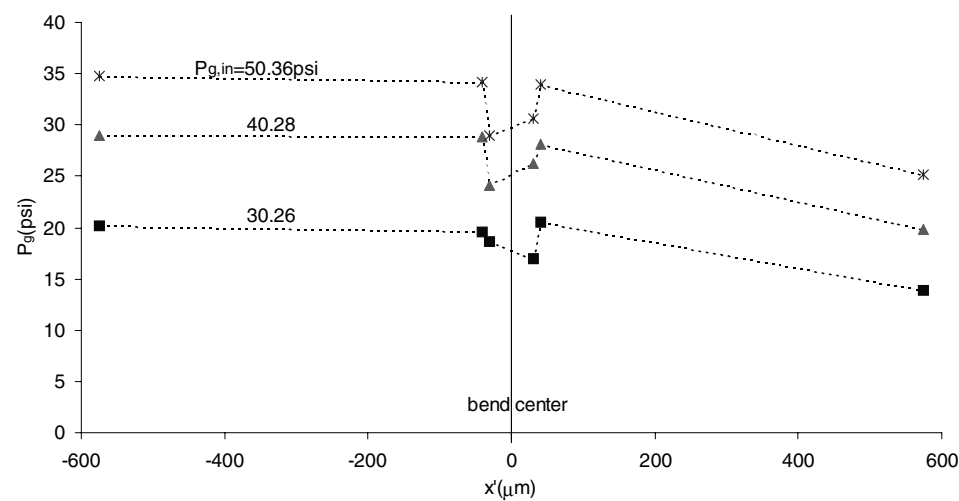

(b)

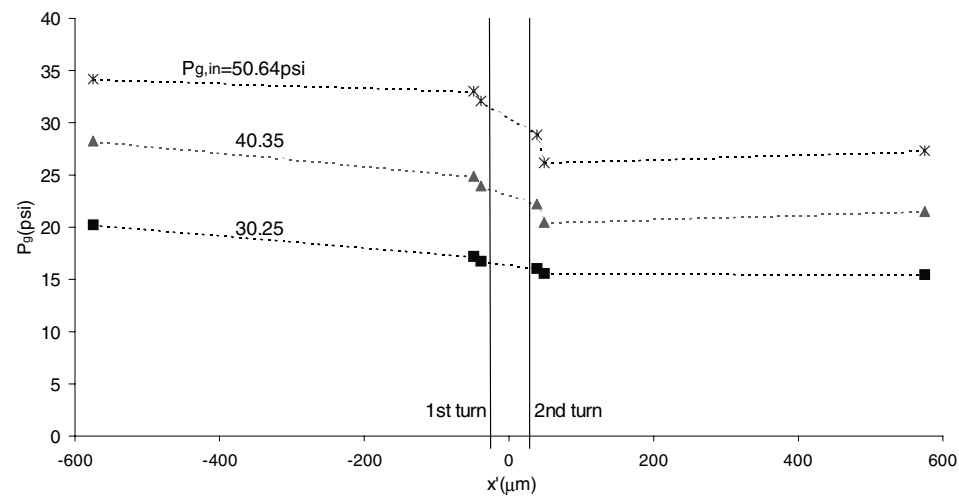

$(c)$

Figure 13. Detailed pressure distributions along the inner wall of the $(a)$ miter, $(b)$ curved, and $(c)$ double-turn bends.

Based on the estimated static pressure either upstream or downstream of the bend, the flow-rate calculations based on equation (1) can be corrected by using either one of the following equations:

$Q_{\mathrm{m}}=\frac{H^{3} W P u_{\mathrm{o}}^{2}}{24 R T(L / 2) \mu}\left[\left(\frac{P_{\mathrm{i}}}{P u_{\mathrm{o}}}\right)^{2}-1+12 K n_{\text {uо }}\left(\frac{P_{\mathrm{i}}}{P u_{\mathrm{o}}}-1\right)\right]$

$Q_{\mathrm{m}}=\frac{H^{3} W P_{\mathrm{o}}^{2}}{24 R T(L / 2) \mu}\left[\left(\frac{P d_{\mathrm{i}}}{P_{\mathrm{o}}}\right)^{2}-1+12 K n_{\mathrm{o}}\left(\frac{P d_{\mathrm{i}}}{P_{\mathrm{o}}}-1\right)\right]$.

The corrected mass flow rates for the microchannels with the three different bends are compared in figure 10 with the measured values. The theoretical predictions are within
$5 \%$ of the experimental measurements. This reasonable agreement confirms that the additional loss cannot be attributed to additional friction loss and is a direct result of the bend.

In fluidic systems, it is very difficult to predict minor losses analytically. Thus, they are usually given in dimensionless form based on experimental data [5]. The most common method used to characterize the loss coefficient is to normalize the pressure loss by the dynamic pressure immediately upstream of the bend. However, surface forces are so dominant in microfluidic systems that the dynamic pressure is, in most cases, very small compared to the driving pressure difference. Therefore, it is more appropriate to normalize the minor pressure loss due to the bend with the major loss due to friction, given in terms of an equivalent length $L_{\mathrm{e}}$. The minor loss $\Delta P_{\mathrm{m}}=P u_{\mathrm{o}}-P d_{\mathrm{i}}$ is expressed in terms of an equivalent length 
of the microchannel that would produce the same pressure loss as the bend. Since the pressure distribution is nonlinear, the equivalent channel length has to be inserted at the location of the bend, as illustrated in figure 11(a). Thus, for any given flow rate through a microchannel with either one of the bends, the pressure upstream and downstream of the bend can be estimated and, subsequently, the equivalent length can be calculated as follows:

$$
Q_{\mathrm{m}}=\frac{H^{3} W P d_{\mathrm{i}}^{2}}{24 R T L_{\mathrm{e}} \mu}\left[\left(\frac{P u_{\mathrm{o}}}{P d_{\mathrm{i}}}\right)^{2}-1+12 K n_{\mathrm{di}}\left(\frac{P u_{\mathrm{o}}}{P d_{\mathrm{i}}}-1\right)\right] \text {. }
$$

$K n_{\mathrm{di}}=P_{\mathrm{o}} K n_{\mathrm{o}} / P d_{\mathrm{i}}$ is the Knudsen number at the downstream section inlet. The results are depicted in figure 11(b), where the equivalent length is almost constant with the flow rate for each bend. The equivalent length for the miter bend is much higher, about $1400 \mu \mathrm{m}$, than the equivalent length for the curved bend, $400 \mu \mathrm{m}$, or the double-turn bend, $100 \mu \mathrm{m}$.

\subsection{Pressure distributions along the bends}

Detailed pressure measurements were conducted along each bend in an effort to understand the influence of the bend on the microchannel flow field. In contrast with the constriction channel [11], which is symmetric with respect to the channel centreline, the channels with bends are not symmetric. Therefore, it is not possible to mix between measurements collected along the inner wall with those along the outer wall in the bending region. In all three devices, shown in figure 2, one sensor was located at the centre of the bend outer wall, symmetric with respect to the distance from the inlet and outlet. This sensor is used as a reference to ensure that the flow forward and backward is identical, not only in terms of the flow rate, but also in terms of the pressure gradient. Two other sensors on the outer wall and three on the inner wall are asymmetric with respect to the bend. Hence, by reversing the flow, five data points can be obtained along the outer wall, as shown in figure 12, and six along the inner wall, as shown in figure 13.

Pressure distributions along the outer wall of the miter, curved and double-turn bends are plotted in figures 12(a)-12(c), respectively, for a variety of flow rate conditions. The distributions along the outer wall of the miter bend are quite flat (figure 12(a)), such that the negative pressure gradient due to friction can be detected neither upstream nor downstream of the bend. Vanishing streamwise pressure gradient in a pressure-driven, viscous flow is usually found in recirculation zones due to flow separation. The classical static pressure peak along the outer wall of a smooth elbow [16], set in as the centripetal force required to turn the flow, can be seen in figure $12(b)$. The pressure distributions along the outer wall of the double-turn bend (figure 12(c)), could be consistent with those of two successive bends with two peaks. However, one sensor did not function and the results are not conclusive.

The corresponding pressure distributions along the inner wall of the miter, curved and double-turn bends are shown in figures 13(a)-13(c), respectively. Upstream of the miter bend (figure 13(a)) the static pressure drops along the flow direction as expected in a viscous flow. However, the pressure just downstream of the bend markedly increases over a short distance and then starts to decrease again. This could be

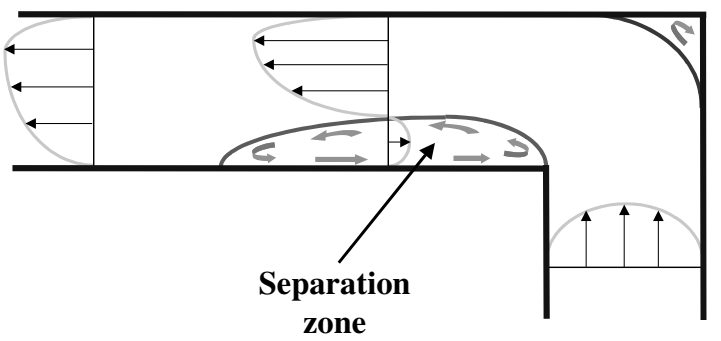

Figure 14. An illustration of possible flow patterns along the miter bend, based on the flow rate and pressure distribution data.

an indication of flow separation due to the sharp corner. Downstream of the re-attachment point, the pressure starts to drop again due to friction. The minimum pressure evident in the curved-bend distributions (figure $13(b)$ ) is consistent with the maximum pressure at the outer wall. The distributions along the double-turn bend (figure 13(c)) could also be interpreted as corresponding to two successive bends.

\section{Flow patterns}

In the present work, the highest mean velocity of the argon gas, $U$, was estimated to be about $0.8 \mathrm{~m} \mathrm{~s}^{-1}$. The corresponding Reynolds number is estimated to be $R e=U D_{\mathrm{h}} / v \sim 0.06$, where $D_{\mathrm{h}}$ and $v$ are the channel hydraulic diameter and gas kinematic viscosity, respectively. Viscous flows with such a low Reynolds number are considered to be creeping flows with no separation. However, if this were the case, not only would the flow rates through all the microchannels with bends be equal to a straight-channel flow rate, but also the pressure gradient would be negative everywhere along the channel. The present results indicate that flow separation may take place on both the inner and outer walls due to the miter bend, as illustrated in figure 14. Energy is needed to maintain such separated flow regions resulting in an additional pressure drop not associated with frictional losses. Hence, the flow rate decreases in comparison with a straight channel without bends. In macrosystems, a swirling secondary flow from the outer to the inner wall occurs because of the imbalance of centripetal forces due to the curvature of the channel centreline. Indeed, guide vanes can be used to eliminate the secondary flow and separation, and thus decrease the pressure loss significantly. However, it is difficult to verify whether a secondary flow also develops in microchannels with bends.

\section{Conclusions}

A series of microchannels with three different bend configurations and integrated pressure sensors has been fabricated and characterized. The measured mass flow rate in all the devices is smaller than that of a straight microchannel with the same length due to the bends. Moreover, although the turn angle is the same for all the devices, $90^{\circ}$, the flow rate is not. The lowest flow rate, about $80 \%$ of the straight channel rate, was measured in the miter-bend microchannel with a sharp corner. The flow rate through the devices with either a curved or double-turn bend was about $95 \%$ of a straight-channel rate. Nonlinear pressure distributions along the straight segments, outside the bend regions, agree 
well with theoretical calculations, and a drop in the pressure upstream and downstream of the bends is observed. This pressure loss increases while the equivalent length is almost constant as a function of the mass flow rate. Detailed pressure measurements around the bend region reveal that the distribution along the miter bend could be the result of flow separation, while the distributions along the curved and doubleturn bends are consistent with fully attached flow in a smooth elbow. Although flow visualizations are not available yet, the possibility of flow separation in a miter-bend microchannel is surprising since the flow in passages with such a narrow gap between the upper and lower surfaces should be fully attached.

\section{Acknowledgment}

This work is supported by the Hong Kong Research Grant Council through RGC grant HKUST6012/98E.

\section{References}

[1] Ho C M and Tai Y C 1998 Micro-electro-mechanical systems (MEMS) and fluid flows Ann. Rev. Fluid Mech. 30 579-612

[2] Van den Berg H R, Seldam C A and Gulik P S 1993 Compressible laminar flow in a capillary J. Fluid Mech. 246 $1-20$

[3] Harley J C, Huang Y, Bau H H and Zemel J N 1995 Gas flow in micro-channels J. Fluid Mech. 284 257-74

[4] Liu J Q, Tai Y C, Pong K C and Ho C M 1993 Micro-machined channel/pressure sensor systems for micro flow studies Proc. 7th Int. Conf. on Solid-State Sensors and Actuators,
Transducers'93 (Japan) pp 995-9

[5] Miller D S 1990 Internal Flow Systems 2nd edn (Cranfield, Bedford: BHR Group Ltd)

[6] Bradshaw P and Wong F Y F 1972 J. Fluid Mech. 52 113-35

[7] Schlichting H 1979 Boundary Layer Theory 7th edn (New York: McGraw-Hill)

[8] Santiago J G, Wereley S T, Meinhart C D, Beebe D J and Adrian R J 1998 A particle image velocimetry system for microfluidics Exp. Fluids 25 316-9

[9] Beskok A, Karniadakis G E and Trimmer W 1996 Rarefaction and compressibility effects in gas microflows J. Fluids Eng. $118448-56$

[10] Li X, Lee W Y, Wong M and Zohar Y 2000 Gas flow in constriction microdevices Sensors Actuators A 83 277-83

[11] Lee WY, Wong M and Zohar Y 2001 Flow separation in constriction microchannels Proc. 14th Int. Micro Electro Mechanical Systems Conf. (MEMS'01) pp 495-8

[12] Lee W Y, Lee S Y K, Wong M and Zohar Y 2000 Microchannels in series with gradual contraction/expansion Proc. Int. Mechanical Engineering Congress and Exposition MEMS vol 2, pp 467-72

[13] Liu J Q, Tai Y C, Lee J J, Pong K C, Zohar Y and Ho C M 1993 In situ monitoring and universal modeling of sacrificial PSG etching using hydrofluoric acid Proc. 6th IEEE Workshop on Micro Electro Mechanical Systems pp 71-6

[14] Wu S, Mai J, Zohar Y, Tai Y C and Ho C M 1998 A suspended microchannel with integrated temperature sensors for high-pressure flow studies Proc. 11th IEEE Workshop on Micro Electro Mechanical Systems (MEMS'98) pp 87-92

[15] Arkilic E B, Schmidt M A and Breuer K S 1997 Gaseous slip flow in long microchannels J. Microelectromech. Syst. 6 $167-78$

[16] Benedict R P 1980 Fundamentals of Pipe Flow (New York: Wiley) 